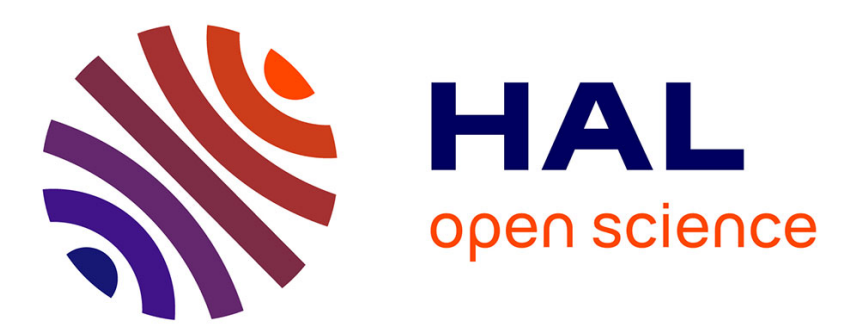

\title{
Territorialité des relations collectives de travail : à propos de l'unité économique et sociale
}

Etienne Pataut

\section{To cite this version:}

Etienne Pataut. Territorialité des relations collectives de travail: à propos de l'unité économique et sociale. Revue Critique de Droit International Privé, 2020, 04, pp.1012. halshs-02517916

\section{HAL Id: halshs-02517916 \\ https://shs.hal.science/halshs-02517916}

Submitted on 13 Jan 2021

HAL is a multi-disciplinary open access archive for the deposit and dissemination of scientific research documents, whether they are published or not. The documents may come from teaching and research institutions in France or abroad, or from public or private research centers.
L'archive ouverte pluridisciplinaire HAL, est destinée au dépôt et à la diffusion de documents scientifiques de niveau recherche, publiés ou non, émanant des établissements d'enseignement et de recherche français ou étrangers, des laboratoires publics ou privés. 


\section{Territorialité des relations collectives de travail : à propos de l'unité économique et sociale}

(Soc, 21 novembre 2018, n¹6-27690, Generali, Dr. Soc. 2019. 141, note C. Radé ; RDT. 2019, pp. 81 et 133 note S. Ranc et Y. Pagnerre et rapp. Joly)

Etienne Pataut

Professeur à l’Ecole de droit de la Sorbonne

Université Paris I Panthéon-Sorbonne

IRJS

Mots Clés : Relations collectives de travail - Représentation des travailleurs - Groupe de sociétés - Unité économique et sociale - Personnalité morale.

Au sein d'un groupe, une unité économique et sociale (UES) peut être reconnue par convention ou par décision de justice entre des entités juridiquement distinctes qu'elles soient ou non dotées de la personnalité morale, dès lors qu'est caractérisée entre ces structures, d'une part, une concentration des pouvoirs de direction à l'intérieur du périmètre considéré ainsi qu'une similarité ou une complémentarité des activités déployées par ces différentes entités, d'autre part, une communauté de travailleurs résultant de leur statut social et de conditions de travail similaires pouvant se traduire en pratique par une certaine mutabilité des salariés

Syndicats c/ Generali

Sur le moyen unique des pourvois principal et incident :

Vu l'article L. 2322-4 du code du travail, alors en vigueur

Attendu qu'au sein d'un groupe, une unité économique et sociale (UES) peut être reconnue par convention ou par décision de justice entre des entités juridiquement distinctes qu'elles soient ou non dotées de la personnalité morale, dès lors qu'est caractérisée entre ces structures, d'une part, une concentration des pouvoirs de direction à l'intérieur du périmètre considéré ainsi qu'une similarité ou une complémentarité des activités déployées par ces différentes entités, d'autre part, une communauté de travailleurs résultant de leur statut social et de conditions de travail similaires pouvant se traduire en pratique par une certaine mutabilité des salariés ;

Attendu, selon l'arrêt attaqué, qu'un accord collectif conclu le 16 novembre 2012 entre des sociétés du groupe Generali assurances et quatre organisations syndicales a redéfini le périmètre de l'UES Generali France assurances, désormais composée des sociétés 
Generali France assurances, Generali vie, Generali IARD, Trieste courtage, Generali réassurance courtage, l'Equité et E-Cie vie, accord actualisé le 26 novembre 2015 du fait de la disparition de la société E-Cie vie, absorbée par la société Generali vie, et de l'entrée dans le périmètre de l'UES de la société Generali France ; que le groupe Generali assurances a mis en œuvre un projet de centralisation et de mutualisation de certaines opérations de gestion des infrastructures informatiques, désormais réunies au sein de la société de droit italien Generali Infrastructure Service (GIS), devenue Generali Shared Services ; que la société GIS a créé le 8 avril 2014 un siège secondaire en France et y a constitué une de ses succursales qui a fait l'objet d'une immatriculation au registre du commerce et des sociétés le 27 mai 2014 ; que la société Generali vie a mis à la disposition de cette succursale cent-soixante-cinq de ses salariés pour une durée de trois ans à effet du 1er juillet 2014 par convention en date du 16 mai 2014 et que chacun des salariés concernés a signé un avenant à son contrat de travail en ce sens ; que, le 22 avril 2015, le syndicat Fédération des employés et cadres Force ouvrière et le syndicat CGT Generali (les syndicats) ont saisi le tribunal d'instance pour l'extension de l'UES existante à la succursale française de la société GIS ;

Attendu que pour rejeter la demande des syndicats, la cour d'appel a retenu qu'il ne peut y avoir d'unité économique et sociale reconnue par convention ou par décision de justice qu'entre des personnes juridiquement distinctes prises dans l'ensemble de leurs établissements et de leurs personnels et qu'il s'ensuit que chacune des personnes juridiquement distinctes composant une unité économique et sociale doit nécessairement être dotée de la personnalité morale, dont l'unité économique et sociale est quant à elle dépourvue, et être ainsi susceptible d'avoir la qualité d'employeur ;

Qu'en se déterminant ainsi, sans rechercher, comme il le lui était demandé, si les salariés employés par la succursale n'étaient pas intégrés à la communauté de travail formée par les salariés de l'UES Generali France assurances, et s'il n'existait pas une unité économique et sociale entre la succursale française de la société italienne en charge des infrastructures du groupe Generali assurances et l'UES Generali France assurances, la cour d'appel n'a pas donné de base légale à sa décision ;

\section{PAR CES MOTIFS :}

CASSE ET ANNULE, mais seulement en ce qu'il rejette la demande d'extension de l'unité économique et sociale Generali France assurances à la succursale française de la société Generali Infrastructure Service, devenue Generali Shared Services, l'arrêt rendu le 20 octobre 2016, entre les parties, par la cour d'appel de Paris ;

Du 21 novembre 2018 - Cour de cassation (Soc.) - Pourvoi $n^{\circ}$ 16-27690 - M. Huglo, prés. - SCP Bouzidi et Bouhanna, SCP Waquet, Farge et Hazan, av.

D'apparence technique et limitée, la décision de la Cour de cassation dans l'affaire Generali est pourtant d'une importance qui explique qu'elle ait immédiatement suscité l'attention des revues spécialisées), que la Cour de cassation ait pris la peine de l'accompagner d'une note explicative sur son site internet et qu'elle soit présentée aux lecteurs de la présente Revue. 
En l'espèce se posait la question du périmètre d'une Unité économique et sociale dans le cadre d'un groupe international, constitué autour de l'assureur Generali.

L'Unité économique et sociale est l'une des grandes innovations techniques du droit du travail pour contourner le cloisonnement d'un groupe de sociétés en personnalités morales différentes. Chaque société est en effet censée mettre en place certaines institutions de représentation du personnel, pour la plupart fusionnées, depuis les ordonnances dites «Macron» de septembre 2017, dans le nouveau Comité social et économique (art. L. 2311-2 et s. C. trav.).

Mais les sociétés s'insèrent parfois dans un ensemble plus vaste, celui du groupe. Aussi, au-delà de cette représentation propre à chaque société, lorsqu'une certaine unité du groupe pouvait se laisser deviner à travers la diversité des personnalités morales, il est apparu nécessaire de prévoir l'organisation de structures de représentation commune. Différents organes ont alors été mis à la disposition des travailleurs, permettant de les représenter dans un ensemble plus vaste que celui de la seule personne morale, notamment le Comité de groupe, aux compétences limitées (art. L. 2332-1 et s. C. trav.) ou le Comité d'entreprise européen (dir. 2009/38 et art. L. 2341-1 et s. C. trav.).

Parfois, l'intégration sociale semble telle qu'il pourrait apparaître nécessaire non pas d'ajouter un organe supplémentaire, comme sont le Comité de groupe ou le Comité d'entreprise européen, mais bien d'englober dans une structure commune plusieurs sociétés dans un organe unique, Comité d'entreprise et, désormais, Comité économique et social.

Aussi le droit du travail met-il à la disposition des travailleurs de ces sociétés la technique de l'Unité économique et sociale qui permet, par accord collectif ou décision de justice, de reconstituer l'unité du groupe au-delà de sa diversité juridique pour des raisons de représentation du personnel en créant un Comité social et économique commun (article L. 2313-18 C. trav.). L'Unité économique et sociale est donc l'une des importantes innovations du droit du travail visant à dépasser le cloisonnement des personnalités morales dans le but de recréer une collectivité de travail (sur l'ensemble, v. p. ex. G. Auzero et al., Droit du travail, 31e éd., Dalloz, 2018, n 1126 et s.). La mise en place de cet organe unique peut toutefois poser quelques difficultés lorsque le groupe n'est pas limité au territoire français.

Tel était le cas en l'espèce. Une Unité économique et sociale avait été reconnue par accord collectif entre différentes sociétés françaises du groupe Generali. Une réorganisation de celui-ci avait toutefois conduit à la centralisation d'un certain nombre d'activités de ce groupe au sein de la succursale française de Generali Italie, succursale à laquelle avaient été affectés 165 salariés de l'une des sociétés françaises. Les syndicats demandaient donc la modification de l'Unité économique et sociale et son extension à la succursale française de la société italienne.

La difficulté venait de ce qu'il était traditionnellement et fermement affirmé qu'une Unité économique et sociale était en principe uniquement composée de personnes juridiques distinctes prises dans l'ensemble de leurs établissements et de leurs personnels, c'est-à-dire entre des entités dotées de la personnalité morale. La solution s'opposait donc à l'intégration dans l'UES de la succursale française d'une personne morale de droit italien.

C'est sur ce point que la Cour de cassation a décidé de faire évoluer son analyse, par un motif finement ciselé : 
«au sein d'un groupe, une unité économique et sociale peut être reconnue par convention ou par décision de justice entre des entités juridiquement distinctes qu'elles soient ou non dotées de la personnalité morale, dès lors qu'est caractérisée entre ces structures, d'une part, une concentration des pouvoirs de direction à l'intérieur du périmètre considéré ainsi qu'une similarité ou une complémentarité des activités déployées par ces différentes entités, d'autre part, une communauté de travailleurs résultant de leur statut social et de conditions de travail similaires pouvant se traduire en pratique par une certaine mutabilité des salariés. »

Désormais, donc, le critère de la personnalité morale n'est plus le critère central, c'est au contraire à la réalité de la constitution d'une communauté de travail qu'il conviendrait de s'attacher. Cette évolution peut sembler de prime abord de détail. Elle constitue pourtant une modification d'importance pour les groupes internationaux de société, permettant l'adaptation de la traditionnelle solution jurisprudentielle issue de l'arrêt "Compagnie des Wagons lits » (I), au point d'ailleurs qu'il est permis de se demander si elle n'incite pas à son dépassement (II).

\section{Adapter « Compagnie des Wagons Lits »}

A. Les lois françaises sur la représentation des salariés seraient des lois de police. La solution est traditionnellement affirmée depuis l'arrêt dit des Wagons-lits, du Conseil d'État (CE, 29 juin 1973, Rev. crit. DIP. 1974. 344, concl. Questiaux et chr. P. Francescakis, p. 273 ; Clunet 1975. 538, note M. Simon-Depitre,) suivi, plusieurs années plus tard, par la Cour de cassation (Soc., 3 mars 1988, Soc. Thoressen Car Ferries Ltd., Rev. Crit. dIP. 1989. 63, note G. Lyon Caen, Clunet 1989. 78, note M.-A. Moreau). Par cette formulation, il faut comprendre, selon les termes de la Cour de cassation que les dispositions françaises s'appliquent à toutes les personnes qui «exercent leur activité en France et qui sont dès lors tenues de mettre en place les institutions qu'elles prévoient ». En d'autres termes, exercer en France la fonction d'employeur, c'est se plier aux lois françaises sur la représentation collective.

Il reste, toutefois, à préciser exactement ce qu'il faut entendre par là. La localisation à l'étranger du siège social de la société peut en effet poser quelques difficultés d'adaptation du droit français, qui en fait le critère d'application des règles en la matière. Aussi est-il généralement admis d'une part que là où la loi française parle de « siège de la société » il faut lire "principal établissement en France » et, d'autre part, que les règles françaises doivent s'appliquer, selon les termes du Conseil d'Etat « à la seule exception de celles qui seraient incompatibles avec la présence à l'étranger du siège social ». La formule ouvre la voie à des adaptations à la fois souples et raisonnables, qui permettent de donner leur plein effet à la loi française en matière de représentation du personnel tout en tenant compte de la réalité du cloisonnement des ordres juridiques.

Cette ligne de solution a ainsi conduit à assouplir la notion complexe d'établissement, la Cour de cassation jugeant que dès lors qu'étaient présents des salariés sur le territoire français, même si ceux-ci étaient peu nombreux et disséminés sur différents sites, il existait "nécessairement » un établissement (Soc, 14 février 2001, Campana CFTC, Dr. Soc. 2001. 639, note M.-A. Moreau). La collectivité de salariés est donc constituée dès que sont exercées en France les prérogatives de l'employeur, quel que soit le mode 
d'organisation choisi et quelle que soit l'institution représentative du personnel en cause (M.A. Moreau, ibid.).

De la même façon, cette souplesse peut conduire à quelques adaptations dans le contentieux électoral. En la matière, en effet, les règles françaises doivent s'adapter non seulement aux différents statuts des salariés (détachés, expatriés, intérimaires...) mais encore à la particularité de l'organisation sociale retenue par l'employeur étranger. En toute hypothèse, en ce qui concerne l'organisation d'élections sur le territoire français, la solution est simple : dès lors que la société emploie des salariés en France, ceux-ci doivent bénéficier d'une représentation collective et, donc, être électeurs, même au prix de quelques adaptations (Soc, 14 janvier 2004, Rev. soc. 2005. 209, note M. Audit).

La souplesse est ici de mise, qui conduit à sauvegarder l'essentiel : la représentation des salariés sur le territoire français.

Il restait, toutefois, à régler le cas particulier de l'Unité économique et sociale et c'est là l'apport de l'arrêt Generali.

B. L'Unité économique et sociale a pour objet de rassembler sous une représentation unique différentes personnes morales. Son objectif est donc de recréer l'unité sociale, au-delà de la diversité des personnalités juridiques. Aussi les critères traditionnels de l'Unité économique et sociale sont-ils, d'une part, la concentration des pouvoirs de l'employeur et la complémentarité des activités et, d'autre part, l'existence d'une communauté de travailleurs. Il s'agit bien, ici, de faire coïncider le fait et le droit et de rechercher, comme soulignait le Président Sargos, cité par le communiqué de la Cour de cassation, « la vérité de la communauté de travail ».

Il reste que cette recherche de la réalité se heurte à deux obstacles d'importance.

Le premier est celui du risque de fractionnement de l'entreprise. L'organisation contemporaine des entreprises est complexe et, surtout, évolutive. Dès lors, la recherche d'une collectivité de travail et d'une unité de direction peut être amenée à changer au cours du temps. Aucun exemple n'en témoigne mieux que la présente espèce, puisque la réorganisation du groupe Generali avait déjà conduit à une modification du périmètre de l'Unité économique et sociale (Soc., 14 novembre 2013, Bull. Civ. 5, n²66), modification à nouveau rendue nécessaire par une nouvelle restructuration du groupe. Le risque est donc qu'à trop vouloir coller à la réalité sociale, la solution perde entièrement toute prévisibilité. D'autre part, si l'Unité économique et sociale vise à coller à la réalité d'une collectivité de travail, il faut aussi veiller à maintenir une unité de représentation au sein de chaque personne morale. Les institutions de représentation du personnel sont créées pour chacune des sociétés ; isoler un établissement au sein d'une société pour le faire régir par des règles particulières au nom de l'autonomie sociale de cet établissement, c'est risquer de porter atteinte aux prérogatives du Comité économique et sociale de la société toute entière.

C'est pour cette raison que, si la Cour a accepté de dépasser le cloisonnement des personnalités morales, elle n'en a pas moins jugé avec constance que les différentes sociétés composant l'entreprise devaient toutes être entièrement intégrées dans l'Unité économique et sociale (Soc, 7 mai 2002, Vivendi, D. 2002. 3119, obs. I. Desbarats, Dr. soc. 2002. 715, note J. Savatier). Il ne saurait être question de procéder à un fractionnement social du groupe, fondé sur la réalité des activités, source inépuisable de contentieux et d'insécurité ; aussi, d'ailleurs, propose-t-on parfois d'abandonner purement et simplement le critère de l'activité, d'un maniement trop délicat (E. Peskine, C. Wolmark, 
Droit du travail, Hypercours Dalloz, 12e éd., 2018, $\left.\mathrm{n}^{\circ} 1114\right)$. La solution traditionnelle de la Cour de cassation permet donc le regroupement de plusieurs personnes morales, sans porter atteinte à l'intégrité sociale de chacune d'entre elles.

Mais à son tour, cette solution se heurte à une seconde difficulté, qui est, cette fois, liée à l'éventuelle internationalité $\mathrm{du}$ groupe. Si une société étrangère possède un établissement sur le territoire français, il est entendu depuis les arrêts Wagons Lits et Thoressen, que celui-ci doit être soumis aux règles françaises sur la représentation collective. Il reste que maintenir la solution traditionnelle posée par l'arrêt Vivendi en présence d'un groupe qui n'aurait qu'une succursale en France, c'est exclure par principe celle-ci de toute Unité économique et sociale. Les salariés de ces établissements sont donc laissés en dehors de toute représentation commune à l'ensemble du groupe.

La solution est d'autant plus contestable que les arguments qui la soutiennent, s'ils peuvent convaincre en droit interne, s'affaiblissent considérablement avec le franchissement de la frontière. Les risques de fractionnement de la représentation collective, en effet, ne sont ici nullement à craindre, puisque, par hypothèse, la personne morale étrangère n'est pas soumise au droit français pour la portion de l'entreprise qui se situe en dehors du territoire français. De ce fait, et contrairement à ce qu'affirmait le pourvoi (dont les arguments sont présentés en détail par le Conseiller Joly, RDT précité, point 4-6), les prérogatives du Comité d'entreprise (désormais du CES) ne peuvent être affaiblies, puisque l'organe de représentation du personnel étranger n'a pas vocation à exercer sa mission sur le territoire français. Permettre l'adjonction dans une UES de l'établissement français d'une société étrangère, c'est, bien au contraire, permettre de tenir compte de la réalité de l'unité de direction et de travail de l'entreprise en France. Les arguments justifiant la solution sont donc bien spécifiques au droit interne.

Aussi, en ce qui concerne ces entreprises internationales (auxquelles la solution nouvelle est probablement limitée, sur ce point, v. C. Radé, note précitée), faut-il se féliciter de son abandon par la Cour de cassation dans l'arrêt Generali. En permettant l'intégration de l'établissement français de la société Generali Italie dans l'UES du groupe Generali en France, la Cour a su exploiter à plein la souplesse ouverte en 1973 par le Conseil d'État et la combiner avec la raison d'être même de l'Unité économique et sociale.

L'UES a pour but de lutter contre le cloisonnement excessif des personnalités morales ; elle permet désormais de s'opposer aussi au second fractionnement qui affecte les sociétés déroulant leurs activités dans l'ordre international : celui des ordres juridiques.

Ne permet-elle pas d'envisager même un dépassement pur et simple de la solution traditionnelle?

\section{Dépasser « Compagnie des wagons lits » ?}

A. Il semble donc acquis que les lois françaises en la matière sont des lois de police. L'arrêt Wagons-lits est l'arrêt sélectionné par les auteurs du célèbre recueil des Grands arrêts de la jurisprudence française de droit international privé (5éd., Dalloz, 2006, $\mathrm{n}^{\circ} 53$ ), pour illustrer la méthode des lois de police et la qualification est explicitement retenue par les juridictions civiles, on l'a vu. 
La solution, pourtant, ne pourrait-elle se discuter? Le droit international privé préconise classiquement le recours à la règle de conflit de lois bilatérale, quitte à corriger le fonctionnement de celle-ci par application de règles impératives du for. De ce fait, dans la théorie dominante, règle de conflit et lois de police vont de pair. Une loi d'une impérativité particulière viendra contrer l'applicabilité d'une règle étrangère désignée par la règle de conflit bilatérale lorsqu'un critère de rattachement spécifique à cette loi impérative se réalise en France. Telle devrait donc être la solution en matière de représentation collective : une règle de conflit bilatérale désignant la loi étrangère ; une loi française impérative avec un domaine propre d'applicabilité. Une telle présentation, pourtant, correspond-elle à la réalité ? Rien n'est moins sûr, tant l'existence même d'une règle de conflit en la matière paraît peu assurée.

L'étude de la jurisprudence montre que le raisonnement est toujours un raisonnement unilatéral, et, surtout, strictement limité aux situations françaises. Non seulement l'application de la loi française est systématiquement justifiée, comme dans l'arrêt Generali, par des critères unilatéralement déterminés, mais encore celle de la loi étrangère (par exemple à des travailleurs hors de France) ne se rencontre jamais. En réalité, la représentation collective paraît échapper purement et simplement au mécanisme conflictuel. Seule compte la loi française, dont le champ d'application international est déterminé par des critères de rattachement qui ne sont pris en considération que s'ils se localisent sur le territoire français. Il y a là les caractéristiques non pas d'une loi de de police, mais bien d'une loi territoriale, au sens le plus fort, qui implique un raisonnement proche du droit public. Si les lois françaises sur la représentation collective sont applicables, c'est en raison de la localisation en France de la situation qu'elles entendent régir et indépendamment de toute loi étrangère qui est purement et simplement exclue du raisonnement (pour une analyse plus détaillée, on se permettra de renvoyer à E. Pataut, "Relire Compagnie des Wagons-lits: La représentation collective des salariés dans l'espace international », in Mélanges Rodière, LGDJ, 2019, p. 387).

Un détour par le droit comparé permettra peut-être de s'en convaincre. Telle est bien, en effet, l'analyse du droit allemand de la cogestion. En application d'une loi de 1976 (Loi sur la codétermination ou Mitbestimmungsgesetz), combinée avec une loi de 2001 (Loi sur la constitution sociale de l'entreprise ou Betriebsverfassungsgesetz), il impose aux sociétés qui emploient plus de 500 salariés de permettre à ceux-ci de désigner un tiers des membres du Conseil de surveillance, voire la moitié au-delà de 2000 salariés. Fréquemment soulignée, la particularité du droit allemand résulte surtout de ce que la participation des salariés se fait directement dans les organes de direction de la société et non seulement par constitution d'organes spécifiques.

La question du champ d'application international de cette loi n'est pas directement réglée par la loi. Elle semble l'être toutefois, dans la doctrine et la jurisprudence majoritaire, précisément par un appel au « principe de territorialité » (V. H. Wißmann, G. Kleinsorge et C. Schubert, Mitbestimmungsrecht : Kommentar, Verlag Franz Vahlen München, $5^{\mathrm{e}}$ éd., 2017, $\S 3$, pt. 26 s.). Concrètement, l'application de ce principe conduit à deux résultats: exclure les salariés en poste à l'étranger dans une filiale ou une succursale de la société allemande du calcul des seuils prévus par la loi, d'une part, exclure ces salariés du corps électoral, d'autre part. En substance, donc, la loi ne concerne que les travailleurs installés sur le territoire national.

Cette solution, qui semble solidement acquise, est justifiée principalement par une raison liée à la souveraineté étatique. L'Allemagne manquerait ainsi de pouvoir ou de 
compétence ( Regelungsmacht») en ce qui concerne les salariés des établissements situés à l'étranger et imposer une telle solution reviendrait donc à violer la souveraineté des pays de travail des salariés en cause. La solution est donc de la façon la plus explicite rapprochée du droit public et des questions de souveraineté (V. H. Wißmann et al., Ibid.).

En Allemagne, donc, les règles en matière de représentation collective ne sont pas seulement des lois de police internationalement impératives. Ce sont de véritables règles territoriales, qui se rapprochent $\mathrm{du}$ droit public et à ce titre entièrement étrangères à la méthode du conflit de lois.

La solution est plus explicitement formulée en Allemagne qu'en France, mais, pour autant, les solutions ne nous semblent pas différentes. Dans les deux cas sont bien présentes les caractéristiques qui permettent de se convaincre de la territorialité d'une loi : l'exclusion de la loi étrangère, d'une part; la seule prise en compte des faits constitués sur le territoire du for, d'autre part. La France et l'Allemagne semblent donc bien avoir la même approche méthodologique des questions de représentation collective dans l'espace.

B. Une telle solution n'est pas sans défaut. En imposant que la loi française s'applique dès que le travail s'exécute en France, elle ne suffit nullement, tout d'abord, à régler la question, le plus souvent passée sous silence, de la représentation collective de travailleurs en poste hors de France. Elle entraîne surtout un morcellement complet des relations collectives de travail, qui sont strictement enserrées dans les limites étatiques.

Bien sûr, la solution de l'arrêt Generali est protectrice, en ce qu'elle a l'avantage de garantir à tous les salariés sur le territoire français la protection de la loi française. Mais elle a pour conséquence corrélative que l'internationalité de la situation est gommée. Imposer l'application de la loi française aux salariés d'une société italienne postés en France, c'est aussi imposer que la représentation des salariés de l'entreprise Generali soit régie par des lois différentes selon leur pays de travail.

Dès lors, ce qu'illustre aussi (et à nouveau) l'arrêt Generali, c'est le contraste frappant et identifié de longue date, entre l'internationalisation du capital et la stricte limite étatique du travail, dont les règles sont fermement maintenues à l'intérieur des frontières nationales. En d'autres termes, l'application de la loi française, qui traduit une certaine résistance à l'internationalisation du droit du travail a un coût, et un coût important, qui est celui du décalage entre un employeur qui prend ses décisions et exerce sa liberté d'action dans un environnement mondial, face à des travailleurs qui, pour leur part, ne peuvent nullement échapper aux règles nationales. Dans cette perspective, la loi nationale peut, certes, être protectrice, mais elle peut tout aussi bien être un carcan ; et lorsque que, comme aujourd'hui, la mondialisation a largement déterritorialisé les entreprises, faire de l'ancrage national de la relation de travail la norme est très problématique (pour une importante réflexion d'ensemble sur ce point v. L. d'Avout, "L'entreprise et les conflits internationaux de lois », Rec. Cours. T. 397).

Le Conseiller Joly souligne à cet égard que la solution pourrait poser des difficultés de droit de l'Union (rapport précité, point 4-5), l'application de la loi française pouvant être analysée comme une entrave aux libertés de circulation qu'il conviendrait dès lors de justifier. La vision parfois restrictive à l'excès de la Cour de justice en la matière pourrait, il est vrai, susciter une certaine inquiétude. On se souvient de l'interprétation excessivement étroite donnée à la justification sociale des entraves par la Cour de justice dans les affaires Laval et Viking (CJCE, 11 décembre 2007, aff. C-488/05 et CJCE, aff. 18 décembre 2007, aff. C-341/05), plus récemment rejointes par une décision qui, à 
nouveau, a procédé à une interprétation très restrictive de la protection due aux salariés et très extensive de la liberté d'entreprendre pour caractériser l'existence d'une entrave (CJUE, Gde Chambre, 21 décembre 2016, aff. C-201/15, AGET Iraklis).

Il reste que, en ce qui concerne la représentation collective, la question est peut-être au moins partiellement tranchée. La difficulté, en effet, a fait en Allemagne l'objet d'un débat important, certains invoquant les risques d'une contrariété avec les exigences de la non-discrimination, d'une part, et de la liberté de circulation des travailleurs, d'autre part, justifiant la saisine de la Cour de justice. Celle-ci, dans un important arrêt Erzberger rendu en Grande chambre, a considéré que la solution allemande ne contrevenait pas aux exigences de l'article 45 TFUE (CJUE, 18 juillet 2017, aff. C-566/15, Konrad Erzberger c/ TUI AG). En substance, la Cour a estimé qu'à défaut d'harmonisation sociale, la liberté de circulation des travailleurs ne garantissait pas une égalité de traitement absolue et indépendante de l'État d'emploi et qu'il n'y avait donc nulle discrimination non plus que nulle entrave à la liberté de circulation dans l'auto-limitation du droit allemand. Le débat est donc clos.

Cette solution, bien sûr, ne peut être transposée sans aucune nuance aux autres libertés de circulation, elle n'en témoigne pas moins d'une relative tolérance du droit de l'Union au mécanisme d'application territorial des règles de représentation collective, qui semblent donc bien avoir vocation à se maintenir, pour le meilleur (la protection des salariés sur le territoire français) et le pire (le morcellement de la représentation collective dans les groupes internationaux).

C. Ce fractionnement peut-il être évité ? La voie semble en tout cas bien étroite. Deux solutions sont envisageables.

La première est celle d'une harmonisation matérielle des règles de représentation collective dans les groupes internationaux. Une telle solution n'est pas inconnue du droit de l'Union, notamment depuis l'institution du Comité d'entreprise européen. Il s'agit sans conteste de la tentative la plus intéressante visant à mettre en œuvre une institution de représentation qui ne soit bornée ni par les personnalités morales ni par les ordres juridiques, même si le Comité d'entreprise européen est encore bien loin d'avoir l'importance de son homologue français. L'harmonisation pourrait aussi se faire par le bas, c'est-à-dire directement au niveau de l'entreprise. Les accords-cadres internationaux sont aujourd'hui une figure importante du droit social international et permettraient sans aucun doute la mise en place de structures de représentation du personnel aux dimensions réelles de l'entreprise et non pas limitées par les frontières des États. Dès lors, l'accord transnational pourrait apparaître comme une voie d'avenir pour intégrer dans l'entreprise ou le groupe d'entreprise en cause des véritables mécanismes de représentation collective. Force est toutefois de constater que ces accords sont encore relativement peu nombreux.

Dès lors, dans l'attente d'une improbable harmonisation matérielle, peut-être pourraiton envisager une seconde solution, celle de l'amélioration de la coordination des règles nationales.

A cet égard, l'insurmontable obstacle de la souveraineté soulevé par la doctrine allemande ne semble en réalité pas infranchissable. L'Avocat Général dans l'affaire Erzberger l'a fort bien dit lorsqu'il a affirmé avec force que :

«l'inclusion dans le régime de codétermination allemand des salariés employés dans d'autres États membres n'impliquerait pas, en tant que telle, une ingérence dans la 
souveraineté ou dans les compétences législatives d'autres États membres. En effet, (...) la question de savoir quels sont les salariés qui peuvent participer aux élections des membres du conseil de surveillance d'une société allemande relève entièrement du pouvoir du législateur allemand. Autrement dit, il n'y a pas de conflit de compétence » (H. Saugmandsgaard Oe, Conclusions précitées, $\mathrm{n}^{\circ} 96$ ).

Dès lors, si la volonté est de représenter une certaine collectivité (les travailleurs rattachés à l'entreprise, les travailleurs rattachés à une unité économique et sociale, les travailleurs rattachés à un établissement...), ne serait-il pas plus pertinent de tenir compte de la réalité de cette collectivité plutôt que de s'arrêter aux frontières étatiques ? Une telle solution conduirait à des rattachements différents en fonction de l'institution de représentation en cause, elle impliquerait aussi que certains salariés se voient appliquer parfois plusieurs lois, par exemple si un salarié d'une entreprise allemande soumise à la codétermination travaille dans un établissement français entrant dans une Unité économique et sociale. Une telle situation ne pose aucune difficulté de principe, le cumul en la matière n'étant nullement signe d'incohérence (en ce sens, v. encore les Conclusions de l'Avocat général Saugmandsgaard Oe, précitées, $n^{\circ} 95$ ).

Il existe même un modèle à suivre en la matière : celui de la sécurité sociale. Partant d'une même situation de stricte territorialité, les règles en matière de sécurité sociale ont progressivement fait une place sinon à la loi en tout cas aux rattachements étrangers. La territorialité, en effet, n'empêche nullement que le critère d'application de la loi soit un peu mieux travaillé pour correspondre aux objectifs substantiels de la règle de fond. Ainsi en matière de sécurité sociale, la loi a-t-elle, par exemple, progressivement supprimé la condition de nationalité comme condition d'accès aux prestations sociales, voire parfois la condition de résidence comme condition de versement des prestations (sur cet exemple, v. P. Rodière, Traité de droit social de l'Union européenne, 2e éd., LGDJ, 2014, pp. 660 et s.).

Surtout, le modèle est transposable à l'Europe toute entière. A nouveau, les règlements en matière de sécurité sociale ont montré qu'il était possible de coordonner les règles nationales grâce à des critères de rattachement communs, même en ne procédant que très exceptionnellement à l'application de la loi étrangère. La réussite spectaculaire de la réglementation européenne vient de la façon dont le champ d'application des lois nationales en matière de sécurité sociale est défini avec précision et, surtout, de façon commune. Les principes, bien évidemment, ne sont pas les mêmes en matière de représentation collective. Il reste que l'exemple du règlement 883/2004 montre bien qu'une détermination européenne des rattachements à mettre en œuvre est possible.

Bien entendu, un important travail d'affinage reste à faire. Il n'en demeure pas moins que, à condition de ne pas s'arrêter à des objections théoriques qui sont autant de tigres de papier, il est parfaitement possible de réfléchir à des critères de rattachement qui permettraient de tenir compte effectivement de la réalité des collectivités de travail susceptibles de faire l'objet d'une représentation dans l'entreprise considérée.

L'arrêt Generali constitue incontestablement un pas dans cette direction. Mettre en place un régime véritablement international de représentation des salariés dans les groupes internationaux supposerait toutefois un saut qualitatif d'une toute autre ampleur. 
